\title{
FORMAÇÃO DE CLASSES DE ESTÍMULOS EQUIVALENTES EM IDOSOS COM IDADE AVANÇADA
}

\section{EQUIVALENCE CLASS FORMATION IN ELDERLY WITH ADVANCED AGE}

\author{
Natalia M. Aggio, Leilane C. K. Antoniazzi, \& Camila Domeniconi \\ UNIVERSIDADE FEDERAL DE SÃO CARLOS, BRASIL
}

\begin{abstract}
RESUMO
O objetivo do presente estudo foi verificar empiricamente a formação de classes equivalentes com participantes com idade avançada utilizando a estrutura de treino CaN e arranjo de treinos e testes do "simples para o complexo". Três idosos, entre 76 e 83 anos, foram expostos ao procedimento de ensino baseado em emparelhamento com o modelo com o propósito de ensinar-lhes novas discriminaçóes condicionais entre estímulos abstratos e depois testar essas relaçóes para averiguar a formação de classes equivalentes. Os resultados demonstraram que os participantes aprenderam as relaçôes condicionais, requerendo um número de repetiçóes dos blocos de treino de linha de base que variou entre 4,83 e 8,37 em média. Além disso, os três participantes apresentaram no teste de equivalência porcentagem de acertos acima de $80 \%$. Dessa forma, foi possível observar sistematicamente a formação de classes de equivalência com participantes idosos usando a estrutura de treino CaN e o arranjo de treinos e testes do "simples para o complexo".
\end{abstract} idosos.

Palavras-chave: equivalência de estímulos, emparelhamento com o modelo, formação de classes equivalentes,

\section{ABSTRACT}

The present study evaluated empirically the formation of equivalence classes with elderly participants using the structure of "comparison-as-node" (CaN) training and the training design of the baseline conditional relations and the probe for emergent relations called "simple to complex". Three participants aged between 76 and 83 years old were exposed to a matching to sample procedure to learn new conditional discriminations between arbitrary stimuli and then such relations were tested to check for the formation of equivalence classes. The results demonstrated that the participants learned the conditional relations, requiring on average between 4.83 and 8.37 repetitions of the baseline training blocks. All participants reached a score of more than $80 \%$ in the equivalence test. Thus it was possible to observe the emergence of equivalence classes with elderly participants using the structure of $\mathrm{CaN}$ training and the training design "simple to complex".

Key words: stimulus equivalence, matching to sample, equivalence class formation, elderly.

A proposição do paradigma de equivalência de estímulos teve início na década de 1970, sendo formalmente concluída na década de $80 \mathrm{com}$ Sidman e colaboradores (Sidman, 1971; Sidman \& Cresson, 1973; Sidman \& Tailby, 1982). Desde então, a formação de classes equivalentes vem sendo alvo de estudos empíricos e conceituais visando ampliar a compreensão de comportamento simbólico e também a proposição de pro- cedimentos de ensino de repertórios complexos para diferentes populações (de Rose, 1993; de Souza \& de Rose, 2000; Duarte \& de Rose, 2007; Galvão, Barros, Goulart, Mendonça \& Rocha, 2002; Medeiros \& Teixeira, 2000).

No estudo inicial, Sidman (1971) trabalhou com um jovem diagnosticado com deficiência mental profunda, que era capaz de selecionar figuras correspondentes a palavras ditadas e

Esta pesquisa contou com apoio financeiro da CAPES, com bolsa de mestrado para a primeira autora e apoio financeiro do PIBIC/CNPq, com bolsa de iniciação científica para a segunda autora. E-mail: camila@power.ufscar.br. 
nomear as figuras. $\mathrm{O}$ autor ensinou este jovem a selecionar palavras impressas correspondentes ao mesmo conjunto de palavras ditadas e após este treino, foram observadas relações não ensinadas diretamente, como a habilidade de relacionar figuras às palavras impressas e vice-versa, bem como a nomeação das palavras impressas. No estudo de 1973, Sidman e Cresson replicaram os procedimentos do estudo anterior com mais dois jovens também com deficiência mental. Foi necessário, no entanto, o ensino de relações adicionais (palavra impressa/palavra impressa e figura/ palavra ditada), mas ao final as mesmas relações emergentes observadas no primeiro experimento foram verificadas para estes participantes. Estes estudos, juntamente com investigações posteriores, levaram à proposição por Sidman e Tailby (1982) do paradigma de equivalência de estímulos. De acordo com esse paradigma, as relações de equivalência seriam relações condicionais entre estímulos que geram outras relações derivadas da recombinação ou produto da substitubilidade dos elementos condicionalmente relacionados. A partir da formação de uma classe de equivalência, os participantes dos estudos citados puderam apresentar leitura com compreensão. Ainda, devido às características de equivalência entre palavras impressas e figuras, a habilidade de nomear figuras foi transferida para as palavras impressas.

A partir dos estudos iniciais, várias outras investigações passaram a ser conduzidas com o objetivo de determinar as condições sob as quais classes de equivalência de estímulos podem ser estabelecidas e mantidas. De um modo geral, os resultados mostraram que o paradigma fornece métodos efetivos e econômicos para o ensino de repertórios complexos (de Souza, Hanna, de Rose, Fonseca, Pereira, \& Sallorenzo, 1997; Hübner \& Marinotti, 2004; Sidman, 1994), uma vez que não requerem o ensino de todas as relações entre os estímulos.
Sabe-se que, apesar do crescimento no número de dados com a temática da equivalência de estímulos que demonstram resultados positivos na formação de classes, ainda existem relatos científicos de variabilidade nos resultados, especialmente em populações com comprometimento de linguagem e em infra humanos (Arntzen \& Holth, 2000; Fields, Hobbie-Reeve, Adams, \& Reeve, 1999; Kato, de Rose \& Faleiros, 2008). Diversas pesquisas têm investigado variáveis que podem influenciar na formação de classes equivalentes, como o tipo de estímulo utilizado, o arranjo do treino e do teste, a estrutura de treino, o número de classes e o tamanho das classes, entre outros (Adams, Fields \& Verhave, 1993; Arntzen \& Holt, 1997; Boelens, 2002; Buffington, Fields, Adams, 1997; Bush, Sidman \& De Rose, 1989; Fields, Varelas, Reeve, Belanich, Wadhwa, Derosse \& Rosen, 2000; Saunders, Drake \& Spradlin, 1999; Saunders \& Green, 1999; Saunders, Wachter \& Spradlin, 1988; Sidman, Kirk \& Willson-Morris, 1985).

Por exemplo, Adams, Fields e Verhave (1993) apontam que quando o arranjo de treino de discriminação condicional é feito do "simples para o complexo", aumentam as chances de que ocorra a formação das classes equivalentes. Neste tipo de arranjo, treina-se uma relação, e logo após o treino, essa relação e sua simetria são testadas. Somente após o teste de linha de base e simetria da primeira relação é que ocorre o treino da segunda relação, que por sua vez é seguido do teste de linha de base e simetria. Os treino e testes continuam nesta seqüência até que as relações de linha de base sejam treinadas e as relações de linha de base e simetria sejam testadas. Assim, no caso de uma classe formada por quatro estímulos, por exemplo, a sequência de treinos e teste seria: treino $\mathrm{BA}$, teste $\mathrm{BA}$ e $\mathrm{AB}$, treino $\mathrm{CA}$, teste $\mathrm{CA}$ e AC, treino DA, teste DA e AD. Somente após esses procedimentos de treino e teste é que as relações equivalentes seriam testadas. 
Alguns estudos apontam que a estrutura de treino pode influenciar o teste de relações equivalentes. O termo estrutura de treino refere-se a seqüência de discriminações condicionais e a forma com que o estímulos são arranjados durante o treino de linha de base (Saunders, \& Green, 1999). Saunders e Green (1999) apontam três tipos de estrutura de treino que podem influenciar nesses testes. São a estrutura de série linear, a estrutura de "umpara-muitos", ou "sample-as-node" (SaN) e a estrutura "muitos-para-um", ou "comparisonas-node" (CaN).

Considerando as diferentes estruturas de treino, Saunders e Green (1999), em um estudo de revisão da área, propuseram que a estrura de treino do tipo "comparison-as-node" (CaN) é a mais efetiva quando comparada às outras estruturas para a obtenção de resultados positivos, em populações com diferentes repertórios, em testes de equivalência. No treino de estrutura $\mathrm{CaN}$, uma classe composta dos estímulos $\mathrm{ABCD}$ seria ensinada na ordem BA, CA, DA. O estímulo A, que neste caso é o estímulo de comparação, se relaciona diretamente com os estímulos B, C e D (modelos) e é chamado de nódulo. É denominado nódulo todo estímulo que se relaciona diretamente, por meio de treino com mais de um estímulo em uma classe (Fields \& Verhave, 1987).

Saunders e Green (1999) sugerem que a diferença verificada ao testar as relações que foram treinadas pelos diferentes tipos de estrutura se deve ao número de discriminações simples requeridas em cada treino e ao número de nódulos envolvidos no treino. As estruturas SaN e $\mathrm{CaN}$ apresentam sempre apenas um nódulo. $\mathrm{Na}$ estrutura $\mathrm{SaN}$ o nódulo é o estímulo modelo, e na CaN o nódulo é o estímulo comparação, porém na estrutura $\mathrm{CaN}$, diferentemente das outras estruturas de treino, todas as discriminações simples requeridas no teste de equivalência são apresen- tadas no treino, o que, portanto, pode facilitar a formação de classes equivalnetes.

Saunders, Drake e Spradlin, (1999) investigaram o efeito da estrutura de treino $\mathrm{CaN}$ e SaN na formação de classes de estímulos equivalentes. Participaram do estudo 11 crianças em idade préescolar que foram distribuídas em dois grupos experimentais. Em um grupo foi utilizada, para o treino de equivalência, a estrutura $\mathrm{SaN}$ e para o outro grupo a estrutura $\mathrm{CaN}$. Os resultados obtidos demonstraram que dos cinco participantes submetidos ao treino com a estrutura $\mathrm{CaN}$, todos obtiveram resultados positivos nos testes de equivalência, enquanto que somente dois dos seis participantes submetidos ao treino com a estrutura SaN obtiveram tal resultado.

Tendo em vista o potencial educacional de procedimentos baseados no paradigma de equivalência de estímulos (Sidman, 1994; de Rose, de Souza, \& Hanna, 1996; de Souza, Hanna, de Rose, Fonseca, Pereira, \& Sallorenzo, 1997) e também a variabilidade nos resultados encontrada em alguns estudos (Arntzen \& Holth, 2000; Fields, Hobbie-Reeve, Adams, \& Reeve, 1999; Kato, de Rose \& Faleiros, 2008), parece pertinente que procedimentos envolvendo a aquisição de repertório socialmente relevante ou envolvendo estimulação de populações com comprometimento cognitivo ou risco de comprometimento, levem em conta a possibilidade de utilizar estruturas de treino que a área considera como menos passíveis de produzir variabilidade, aumentando as probabilidades de sucesso do procedimento.

$\mathrm{Na}$ literatura sobre envelhecimento são encontrados vários estudos correlacionando o avanço da idade com maiores dificuldades na aprendizagem e manutenção de novos repertórios e outros comprometimentos cognitivos (Baron \& Menich, 1985; Pérez-González \& Moreno-Sierra, 1999; Pinto, 1999). A despeito do aumento do número de pessoas idosas no 
Brasil, pesquisas utilizando o paradigma de equivalência de estímulos carecem de dados com essa população. Pérez-González e Moreno-Sierra (1999) publicaram um dos poucos estudos com o paradigma da equivalência de estímulos com idosos. O propósito desse estudo foi investigar a formação de classes de equivalência em idosos, comparando os resultados deles com os dos participantes mais jovens. No Experimento 1, participaram um adolescente de 13 anos, três adultos jovens com 21, 44 e 53 anos, e quatro idosos, com 66, 67, 70 e 74 anos; a estrutura de treino era série linear $(A-B-C)$. Todos os participantes formaram classes de estímulos equivalentes. De acordo com os autores, as fases de testes foram conduzidas até o participante atingir o critério de seis tentativas consecutivas corretas nos testes de discriminação condicional. Segundo os mesmos, há alguma probabilidade de que repetir o mesmo tipo de tentativa na fase de teste pode promover algum feedback da resposta correta em pessoas que falharam nas tentativas iniciais. Além disso, verificou-se que havia erros nas relações de linha de base durante a fase de teste. Assim, foi realizado o Experimento 2 com o intuito de promover uma quantidade maior de treino antes do teste e uma quantidade fixa de tentativas na fase de teste. Nesse segundo experimento, participaram dois idosos, um com 65 e outro com 73 anos. O procedimento específico utilizado no Experimento 2 para diminuir a quantidade de erros não foi eficaz, visto que os dois participantes tiveram uma quantidade de erros parecidos dos participantes do Experimento 1.

Nos estudos de Pérez-González e MorenoSierra (1999), os seis participantes idosos aprenderam as discriminações condicionais de linha de base e formaram classes de estímulos equivalentes. Entretanto, houve uma diferença clara entre o desempenho dos idosos quando comparado com o desempenho dos participantes mais jovens, visto que estes últimos apresentaram poucos erros nas fases de treino e de teste. De uma forma geral, os resultados com os idosos sugerem que eles aprendem novas relações entre novos estímulos com mais dificuldade que os adultos jovens, mas, uma vez aprendida as discriminações de linha de base, as relações de equivalência emergiram. Pérez-González e Moreno-Sierra (1999) sugerem que estudos com a temática da equivalência de estímulo podem ser úteis para o planejamento de diferentes procedimentos de ensino e inclusive para estimulação da memória em pessoas idosas.

Segundo Pérez-González e col. (1999), estudos sobre memória e inteligência com pessoas idosas demonstram que essa população pode apresentar dificuldades em lembrar novas informações e resolver novos problemas. Entretanto, quando lidam com tarefas diretamente relacionadas com fatos que lhe são familiares, os idosos demonstram um desempenho similar aos de pessoas mais jovens. Procedimentos baseados no paradigma da equivalência de estímulos tem sido utilizados no ensino eficiente de repertórios complexos para diferentes populações (de Souza, Hanna, de Rose, Fonseca, Pereira, \& Sallorenzo, 1997; Hübner \& Marinotti, 2004; Sidman, 1994), mas ainda são escassos os estudos com a população idosa.

O objetivo do presente estudo foi verificar empiricamente a formação de classes equivalentes com participantes com idade avançada utilizando a estrutura CaN e arranjo do "simples para o complexo". O presente estudo pode ser um primeiro passo para investigações posteriores que visem explicitar sistematicamente quais variáveis são pertinentes para formação de classes por idosos e, assim, subsidiar a programação de ensino de conteúdos simbólicos para essa população a fim de estimular a aprendizagem e manutenção de novos repertórios e retardar possíveis efeitos deletérios do envelhecimento relacionados com perdas cognitivas. 


\section{MÉTodo}

\section{Sujeitos}

Participaram da pesquisa três idosos com 76, 82 e 83 anos que não apresentavam perdas cognitivas. Para avaliação de perdas cognitivas foi usado o instrumento "Mini-exame do estado mental" (Folstein, Folstein \& McHugh, 1975). A pontuação do instrumento é feita com base na escolaridade dos participantes. A Tabela 1 apresenta os participantes, a idade e a escolaridade de cada um, assim como a pontuação no "Mini-exame do estado mental". A tabela também fornece informação sobre o número de pontos mínimo esperado para que o participante seja considerado sem perdas cognitivas. Os resultados no "Mini-exame do estado mental" (MEEM) apontaram que os participantes provavelmente não apresentavam perdas cognitivas.

O procedimento obteve o aceite do comitê de ética em pesquisa com seres humanos da UFSCar, parecer número 265/2008 e todos os participantes leram e assinaram o Termo de Consentimento Livre e Esclarecido.

\section{Tabela 1}

Características dos participantes (idade, escolaridade e pontuação no "Mini-exame do estado mental” - MEEM) e pontuação mínima esperada pela escala do MEEM.

Participantes* Idade Escolaridade Pontuação Pontuação mínima esperada

\begin{tabular}{lllll}
\hline Noeli & 76 & Até $^{\text {a }}$-EF** & 27 & 24 \\
Tomas & 83 & Sup. Comp & 26 & 26 \\
Zumira & 82 & Até 4 $4^{\mathrm{a}}-\mathrm{EF}$ & 24 & 24 \\
\hline
\end{tabular}

*nomes fictícios.

$* *(\mathrm{EF}=$ Ensino Fundamental $)$.

\section{Equipamentos}

Para o desenvolvimento da pesquisa foi utilizado um microcomputador Apple Macin- tosh Performa 6360. Foi empregado o software MTS versão 11.6 (Dube \& Hiris, 1996), que permite a programação de tarefas de matchingto-sample, para o gerenciamento da aplicação do procedimento experimental e o registro de dados.

\section{Estimulos}

No pré-treino, os estímulos foram figuras familiares (animais, frutas e flores) bidimensionais coloridas. $\mathrm{Na}$ fase de treino, os estímulos eram figuras abstratas tridimensionais coloridas. Todas as figuras eram acopladas ao próprio software MTS. A Tabela 2 mostra os estímulos não familiares usados. Os números indicam as classes de estímulos a serem formadas e as letras, o conjunto de estímulos que compõem as classes.

\section{Tabela 2}

Estímulos utilizados nos procedimentos de treinos e testes, e suas respectivas classes.

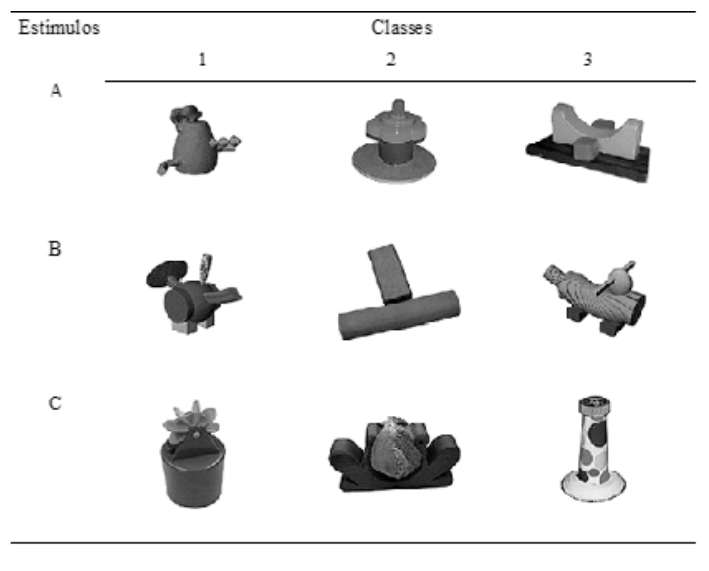

\section{Procedimento}

A duração das sessões variava entre 30 e 40 minutos de acordo com a disposição do participante. As sessões eram diárias e ocorriam na residência dos próprios participantes, podendo ser realizadas mais de uma sessão por dia, de acordo com a disposição do participante. Caso a experimentadora notasse sinais de cansaço a sessão era interrompida. Ao término de cada 
sessão a experimentadora procurava conversar sobre o dia-a-dia dos participantes a fim de tornar a participação na pesquisa mais reforçadora.

Foram ensinadas três classes de estímulos com três estímulos em cada classe em treinos de discriminação visual-visual com as figuras abstratas representadas na Tabela 2. Todos os blocos de treino e teste foram apresentados com a estrutura $\mathrm{CaN}$, ou seja, o estímulo comparação era o nódulo e permanecia constante nos treinos, mudando sucessivamente os estímulos modelo. As tentativas de treino consistiam nos estímulos A como comparação e os demais estímulos (B e C) como modelo nos seus respectivos treinos.

Nos blocos de treino e teste o critério de aprendizagem era de 90\% de acertos no bloco e 90\% de acertos em cada uma das relações, com exceção do Teste de Equivalência, que não possuía critério de aprendizagem. Por exemplo, num bloco com 18 tentativas, sendo seis tentativas B1A1, seis B2A2 e seis B3A3, o participante poderia errar no máximo duas tentativas no geral sendo que o número de erros em cada relação (B1A1, B2A2 e B3A3) não poderia ser superior a um erro.

Pré-treino. O Pré-treino tinha como objetivo ensinar aos participantes como proceder nas tarefas de discriminação condicional. Foram realizadas 18 tentativas de escolha de acordo com o modelo com relações entre estímulos-modelo visuais e comparações visuais, em que havia três escolhas em cada uma. A tarefa consistia em relacionar de forma arbitrária figuras familiares (animais, frutas e flores). A seleção do estímulo de comparação correto produzia uma tela com retângulos branco e preto que alternavam de cor, e a seleção incorreta resultava em uma tela preta.

Na primeira tentativa, a experimentadora dava as instruções de como realizar as tarefas. Ela instruía o participante a clicar com o mouse sobre a figura central (estímulo-modelo). Após essa resposta, três figuras laterais apareciam na tela (estímulos de comparação), então o participante era instruído a escolher e clicar em apenas uma das figuras e era orientado a observar a conseqüência da resposta, ou seja, a experimentadora explicava que a tela com retângulos branco e preto que alternavam de cor significava que a resposta tinha sido correta, e a tela preta significava que a resposta dada anteriormente estava errada.

Treino de Discriminação Condicional BA. Nesta fase os participantes aprenderam a relação: BA (B1A1, B2A2, B3A3). O treino de discriminação condicional de cada relação era feito em dois blocos. No primeiro bloco, com 100\% de conseqüências e 21 tentativas, foram inseridos gradualmente os estímulos de comparação. Assim, era apresentada primeiramente a relação B1A1, sendo que apenas o estímulo de comparação A1 estava disponível para escolha pelo participante na primeira tentativa. $\mathrm{Na}$ segunda tentativa era apresentada a relação B2A2 e os estímulos de comparações eram A1 e A2. $\mathrm{Na}$ terceira tentativa era apresentada a relação B3A3 e os estímulos de comparações eram A1, A2 e A3. A partir da quarta tentativa eram apresentadas três tentativas consecutivas da mesma relação com os três estímulos de comparação. Por fim, as relações eram randomizadas, controladas as posições e o número de vezes que cada estímulo aparecia em uma posição. As tentativas tinham conseqüências diferenciais iguais às da fase de pré-treino. Se o participante atingisse o critério de acertos passava para o Bloco 2, caso contrário o Bloco 1 era repetido. O Bloco 2 apresentava as mesmas relações que o anterior, com 18 tentativas randomizadas e com $50 \%$ de conseqüências. A diminuição na probabilidade de reforçamento teve intuito de preparar o participante para as tentativas de teste, nas quais não existe nenhuma conseqüência 
diferencial para acerto ou erro. Se o participante não atingisse o critério no Bloco 2, o Bloco 1 era repetido, seguido do Bloco 2.

Testes de Linha de Base e de Simetria $\mathrm{BA} / \mathrm{AB}$. Após passar pelo treino discriminativo da relação BA, o participante realizava dois blocos de Teste de Linha de Base e Simetria (um com 36 e outro com 54 tentativas). Os dois blocos foram constituídos pelos mesmos tipos de tentativas e verificavam o desempenho nas mesmas relações, eles foram divididos apenas para não ficarem muito longos. Se não atingisse o critério de acertos, o participante retornava para o Bloco 2 de treino, no qual ocorria o treino dessa relação com $50 \%$ de conseqüências diferenciais programadas para acertos e erros. Nesta etapa não havia conseqüências diferenciais programadas para acertos e erros.

Ao atingir o critério estabelecido (90\% de acertos em cada uma das relações testadas) nestes testes, a relação CA era treinada (C1A1, C2A2, C3A3).

Treino de Discriminação Condicional CA. O treino CA obedeceu a mesma seqüência de passos e critérios descritos anteriormente, no treino da relação BA. Também foi dividido em dois blocos, com 21 e 18 tentativas cada um.

Testes de Linha de Base e de Simetria CA/AC. O teste ocorreu seguindo a mesma seqüência do teste das relações BA e AB.

Treino de Linha de Base Cheia. Este treino era feito após o término de todos os blocos de treino e teste de linha de base e simetria e imediatamente antes do teste de equivalência. Foram treinadas todas as relações de linha de base em um bloco programado com conseqüências diferenciais em 50\% das tentativas e contendo 36 tentativas em cada bloco. Se o participante atingisse o critério de acertos, passava para a etapa seguinte, caso contrário, repetia o bloco de treino de Linha de Base Cheia. Caso não atingisse o critério no bloco de Linha de Base Cheia este bloco era repetido por no máximo quatro vezes. Se ainda assim o participante não atingisse o critério era repetido o bloco de treino em que se concentravam as relações em que não foi atingido o critério.

Teste de Equivalência ABC. O teste das relações equivalentes era feito após a conclusão do Treino de Linha de Base Cheia e foi composto por 54 tentativas. Nele eram apresentadas todas as relações treinadas e testadas $(\mathrm{BA}, \mathrm{AB}$, CA, AC, BC e CB). Não havia nenhum tipo de conseqüência diferencial nessa etapa.

A Tabela 3 resume a seqüência de treino e testes adotada, as relações que foram ensinadas, o número de blocos, o número de tentativas em cada bloco e a porcentagem de conseqüências nos blocos de treino.

\section{Tabela 3}

Seqüência de treino e testes, relações envolvidas e número de tentativas nos blocos. Entre parênteses a porcentagem de conseqüências em cada uma das etapas de treino e teste. (Teste de LB e S = Teste de Linha de Base e Teste de Simetria; Tent $=$ Quantidade de Tentativas Programadas).

\begin{tabular}{lccc}
\hline Procedimento & Relações & Blocos & Tent \\
\hline Treino $(100 \%)$ & BA & 1 & 21 \\
Treino $(50 \%)$ & BA & 2 & 18 \\
Teste de LB e S $(0 \%)$ & BA e AB & 3 & 36 \\
Teste de LB e S $(0 \%)$ & BA e AB & 4 & 54 \\
Treino $(100 \%)$ & CA & 5 & 21 \\
Treino (50\%) & CA & 6 & 18 \\
Teste de LB e S $(0 \%)$ & CA e AC & 7 & 36 \\
Teste de LB e S $(0 \%)$ & CA e AC & 8 & 54 \\
LB Cheia (50\%) & BA/CA & 9 & 36 \\
Teste misto $(0 \%)$ & ABC & 10 & 54 \\
\hline
\end{tabular}

\section{RESULTADOS}

Serão apresentados os resultados relativos ao desempenho dos sujeitos durante as fases 

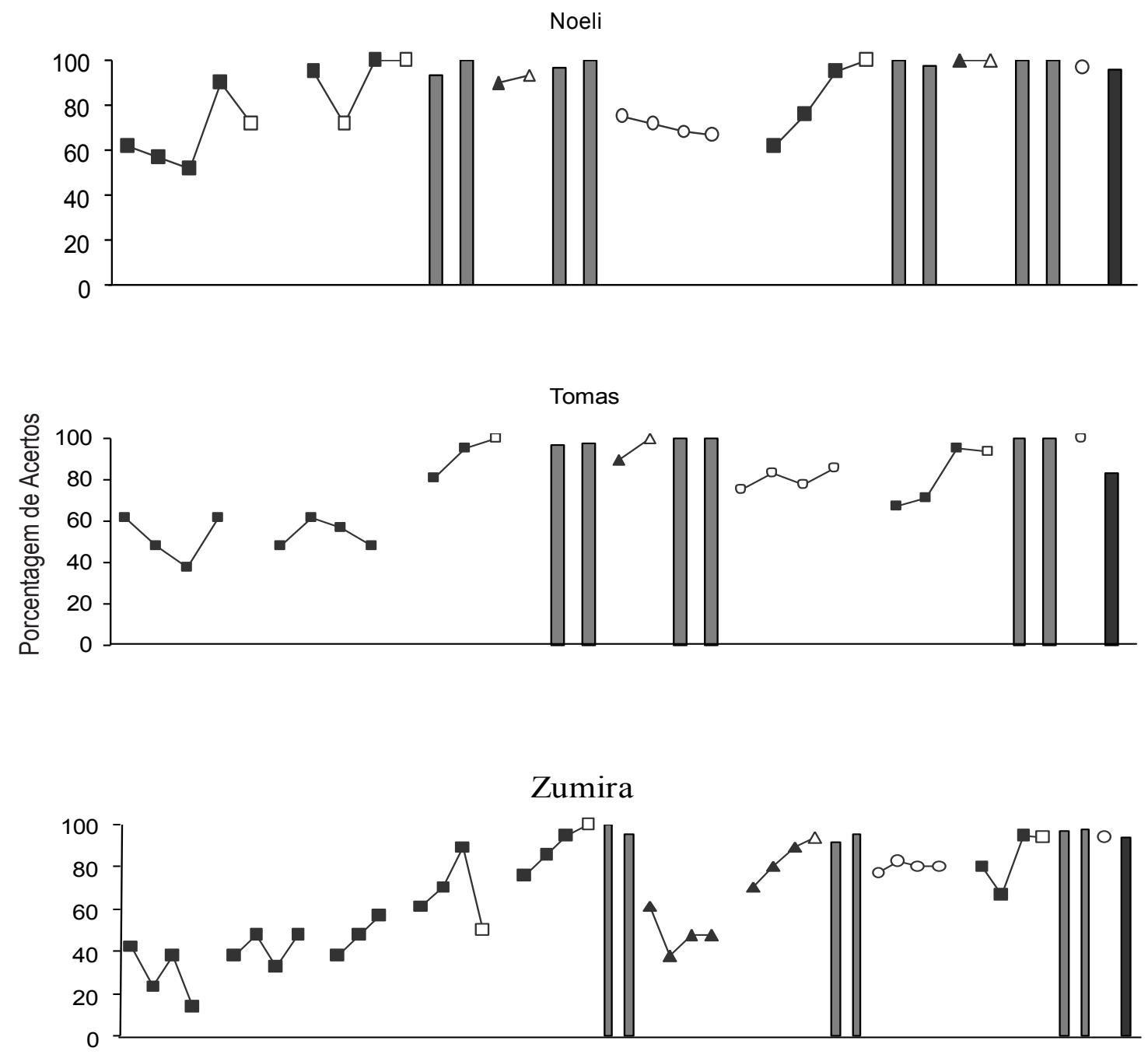

Fases de Treino e Teste

Figura 1. Porcentagem de acertos de cada participante nas fases de treino e teste. Os retângulos cheios representam o treino BA com 100\% de feedback; os retângulos vazios representam o treino BA 50\% de feedback; os triângulos cheios, o treino CA 100\% de feedback; os triângulos vazios, o treino CA 50\% de feedback. Os círculos vazios representam os Treinos de Linha de Base Cheia. As barras cinza se referem aos Testes de Linha de Base e Simetria e as pretas aos Testes de Equivalência. O rompimento da linha que une os blocos de um mesmo passo significa que houve uma pausa entre os blocos.

de treino BA, CA; Testes de Linha de Base e Simetria BA/AB e CA/AC; e Teste de Equivalência $A B C$. A Figura 1 mostra a porcentagem de acertos de cada participante em todos os blocos de treino e teste. Na Tabela 4 estão detalhados resultados de treinos e testes.

Observa-se a partir dos dados apresentados na Figura 1 um maior número de repetições na primeira relação ensinada (BA) quando comparada ao número de repetições da segunda (CA) para todos os participantes. Os três participantes não atingiram critério nas primeiras exposições aos blocos de linha de base cheia, tendo que pelo menos repetir o treino da relação BA (a participante Noeli também refez o treino da relação CA). Em relação ao Teste de Equivalência, todos os par- 
ticipantes apresentaram porcentagem de acertos acima de $80 \%$. O participante Tomas apresentou 86\% de acertos, Noeli 96\% e Zumira 94\%.

A Tabela 4 mostra o índice de repetição de cada participante em cada fase de treino e o número total de tentativas de treino requeridas até que o critério fosse atingido. $\mathrm{O}$ índice foi calculado dividindo o número total de tentativas de treinos realizados pelo número mínimo exigido.

\section{Tabela 4}

Índice de repetição (IR) e número total de tentativas (TT) de treino das relações BA e CA. Os dados aparecem divididos em Treino BA, Treino CA, Linha de Base Cheia, média do índice de repetição e total do número de tentativas de todos os treinos.

\begin{tabular}{|c|c|c|c|c|c|c|c|c|}
\hline \multirow[t]{2}{*}{ Participante } & \multicolumn{2}{|c|}{$\begin{array}{l}\text { Treino } \\
\text { BA }\end{array}$} & \multicolumn{2}{|c|}{$\begin{array}{l}\text { Treino } \\
\text { CA }\end{array}$} & \multicolumn{2}{|c|}{$\begin{array}{l}\text { Linha } \\
\text { de Base } \\
\text { Cheia }\end{array}$} & \multirow{2}{*}{$\begin{array}{l}\text { Média } \\
\text { Índice } \\
\text { Repet. } \\
\text { X }\end{array}$} & \multirow{2}{*}{$\begin{array}{l}\text { Total } \\
\mathrm{N}^{\mathrm{o}} \text { de } \\
\text { Tentat. } \\
\mathrm{X}\end{array}$} \\
\hline & IR & TT* & IR & TT & IR & $\mathrm{TT}$ & & \\
\hline Joeli & 7,5 & 225 & 2 & 60 & 5 & 90 & 4,83 & 375 \\
\hline Tomas & 9,7 & 291 & 1 & 30 & 5 & 90 & 5,23 & 411 \\
\hline Zumira & 14,9 & 447 & 5,2 & 156 & 5 & 90 & 8,37 & 693 \\
\hline
\end{tabular}

* Foram consideradas apenas as tentativas com conseqüência diferencial, deste modo metade das tentativas dos blocos de $50 \%$ de feedback foram excluídas.

É possível observar na Tabela 4 que o índice de repetição variou entre 7,5 e 14,9 no treino das relações BA e entre 1 e 5,2 no treino das relações CA. Os blocos de Linha de Base cheia que incluem tanto tentativas de treino BA como CA foram repetidos 5 vezes por todos os participantes. A média geral do índice de repetição variou entre 4,83 e 8,37.

\section{DISCUSSÃO}

O estudo apresentou o desempenho de participantes com mais de 75 anos em tarefas de formação de classes equivalentes. Os resul- tados apontaram que os participantes repetiram os blocos de treino entre 4,83 e 8,37 vezes até que o critério fosse atingido. Sobre os resultados em testes, foi observada a emergência de relações equivalentes logo no primeiro teste.

O procedimento aplicado utilizou variáveis apontadas na literatura como facilitadoras do treino e da emergência de relações de equivalência. As variáveis foram a estrutura de treino $(\mathrm{CaN})$ e a seqüência de aprendizagem, do "simples para o complexo". Essas variáveis combinadas já se mostraram eficazes em estudos com outras populações como crianças (Omote, Vicente, Aggio \& Haydu, 2009; Rocha, 2002) e universitários (Haydu \& de Paula, 2008; Haydu, Omote, Vicente, \& Aggio, submetido). O presente estudo mostrou sua efetividade também com idosos de idade avançada, mostrando o potencial desse sistema de treino de discriminações condicionais para a aprendizagem dessas e emergência de relações não diretamente ensinadas.

Para esclarecimentos sobre qual dessas variáveis pode ter sido crucial na produção dos resultados encontrados são necessárias investigações adicionais, nas quais seja manipulada uma variável de cada vez e comparados desempenhos de idosos em delineamentos de grupo ou de sujeito único.

Apesar de haver diferenças nos procedimentos do presente estudo e do estudo de Pérez-González e Moreno-Sierra (1999), o qual teve como objetivo comparar o desempenho de participantes de diferentes faixas etárias, incluindo idosos, na formação de classes, algumas comparações podem ser feitas em relação ao índice de repetição de tentativas de treino. O índice de repetição é calculado dividindo o número mínimo de repetições requeridas pelo número real feito pelo participante. Enquanto no presente estudo o índice foi de 5,43 para o participante Tomás, 4,83 para a participante 
Noeli e 8,37 para a participante Zumira, no estudo de Pérez-González e Moreno-Sierra (1999) este índice foi de 2,6, 6,83 e 23,66 para os três participantes mais velhos. Esses resultados confirmam a eficácia do procedimento. Outro dado importante é o de que os participantes formaram relações equivalentes logo no primeiro teste. A idade dos participantes no presente estudo confere novidade ao dado, visto que os participantes têm idade significativamente mais avançada que os de Pérez-González e MorenoSierra (1999).

No presente estudo todos os participantes apresentaram emergência das relações equivalentes, porém houve diferenças nos desempenhos dos três participantes nas fases de treino, em especial em relação à participante Zumira, que requereu um número maior de blocos de treino para que o critério fosse atingido do que os outros participantes. Podem ser levantadas algumas hipóteses que expliquem este fato, como o valor reforçador da tarefa, os diferentes controles de estímulos presentes ou as experiências prévias de cada participante. Investigações posteriores são necessárias para que possa ser feita uma análise mais precisa sobre as variáveis que influenciam na variabilidade dos resultados.

O estudo de procedimentos de ensino que se mostrem eficazes com a população idosa se faz mais importante, uma vez que esta é uma faixa etária em crescimento em todo mundo. Perdas cognitivas fazem parte do processo de envelhecimento saudável (Yassuda, Batistone, Fortes \& Néri, 2006), porém variáveis biológicas não são por si só determinantes dessas perdas. Baron e Menich (1985) e Baron e Surdy (1990) afirmam que essas dificuldades são resultantes de uma interação entre mudanças no sistema nervoso e estimulação ambiental, e desse modo um ambiente com pouca estimulação também interfere nessas perdas.
O estudo realizado por Baron e Menich (1985) avaliou o desempenho de jovens e idosos em tarefas de discriminação condicional e o papel do treino no aprimoramento da execução dessas tarefas nas duas faixas etárias. Jovens e idosos passaram por um treino de discriminação condicional onde o tempo limite para responder foi manipulado. Os resultados mostraram um melhor desempenho dos participantes jovens, porém apontaram que o treino favoreceu o desempenho tanto dos jovens quantos dos idosos, mostrando a efetividade do treino em melhorar o desempenho também da população com mais idade.

Esses resultados mostraram a importância do estudo das variáveis relacionadas com a aprendizagem e manutenção de conteúdos em idosos, uma vez que apontam que a aprendizagem e recordação estão ligadas também à maneira como o ambiente é arranjado.

Uma vez que procedimentos de ensino baseados no paradigma de equivalência de estímulos têm sido apontados como efetivos e econômicos para o ensino de repertórios complexos (de Souza, Hanna, de Rose, Fonseca, Pereira, \& Sallorenzo, 1997; Hübner, \& Marinotti, 2004; Sidman, 1994), faz-se importante investigações também com idosos, já que esta é uma população que apresenta demanda de estimulação cognitiva. Pesquisas básicas que investiguem os processos envolvidos no controle de estímulos com participantes idosos são um primeiro passo no desenvolvimento de tecnologias comportamentais que beneficiem esta população.Os desempenhos observados nas fases de treino e teste mostraram a eficácia do procedimento no ensino de participantes com idade avançada e corroboram para os estudos na área de equivalência de estímulos. 


\section{REFERÊNCIAS}

Adams, B. J., Fields, L., Verhave, T. (1993). Effects of test order on intersubject variability during equivalence class formation. The Psychological Record, 43, 133-152.

Arntzen, E., \& Holth, P. (1997). Probability of stimulus equivalence as a function of training design. The Psychological Record, 47, 309-320.

Arntzen, E., \& Holth, P. (2000). Probability of stimulus equivalence as a function of class size vs. number of classes. The Psychological Record, 50, 79-104.

Baron, A. \& Menich, S. R. (1985). Reaction times of younger and older men: effects of compound samples and prochoice signal on delayed matching-to-sample performances. Journal of the Experimental Analysis of Behavior, 44, 1-14.

Baron, A., \& Surdy, T. M. (1990). Recognition memory in older adults: adjustment to changing contingencies. Journal of the Experimental Analysis of Behavior, 54, 201-212.

Bush, M. K., Sidman, M., \& de Rose, T. (1989). Contextual control of emergent equivalence relations. Journal of the Experimental Analysis of Behavior, 51, 29-45.

Boelens, H. (2002) .Studying stimulus equivalence: defense of the two-choice procedure. The Psychological Record, 52, 305-314.

Buffington, D. M, Fields, L., Adams, B. J. (1997). Enhancing equivalence class formation by pre-training of other equivalence classes. The Psychological Record, 47, 69-96.

de Rose, J. C. (1993). Classes de estímulos: implicações para uma análise comportamental da cognição. Psicologia: Teoria e Pesquisa, 9, 283-303.

de Souza, D. \& de Rose, J. (2000). Reading, equivalence and recombination of units: A replication which students which different learning histories. Journal of Experimental Analysis of Behavior, 33, 97-100.

de Souza, D. G. , Hanna, E. S. , de Rose, J. C. , Fonseca, M. L. , Pereira, A. B., Sallorenzo, L. H.
(1997). Transferência de controle de estímulos de figuras para texto no desenvolvimento de leitura generalizada. Temas em Psicologia (Ribeirão Preto), 1, 33-46.

Duarte, G. M., \& de Rose, J. C. (2007). A aprendizagem simbólica em crianças com déficit atencional. Revista Brasileira de Educaşão Especial, 12, 331-350.

Dube, W. V., \& Hiris, E. J. (E. K. Shriver Center for Mental Retardation, Inc): MTS v 11.6. Software desenvolvido somente para Pesquisa e Educação, 1996.

Fields, L., Hobbie-Reeve, S. A., Adams, B. J., \& Reeve, K. F. (1999). Effects of training directionality and class size on equivalence class formation by adults. The Psychological Record, 49, 703-724.

Fields, L., Varelas, A, Reeve, K. F., Belanich, J., Wadhwa, P., Derosse, P, Rosen, D. (2000) .Effects of prior conditional discrimination training, symmetry, transitivity, and equivalence testing on the emergence of new equivalence classes. The Psychological Record, 50, 443-466.

Fields, L., \& Verhave, T. (1987). The structure of equivalence classes. Journal of the Experimental Analysis of Behavior, 48, 317-332.

Folstein, M. F., Folstein, S. E. \& McHugh, P. R. (1975). Mini-mental state: a practical method for grading the cognitive state of patients for the clinician. J. Psychiatri Res, 12, 189-98.

Galvão, O. F. Barros, R. S Goulart, P. R. K., Mendonça, M. B., Rocha, A. C. (2002). Escola experimental de primatas. Estudos de Psicologia (Natal), 7(2), 361-370.

Haydu, V. B., \& de Paula, J. B. C. (2008). Estabilidade de classes equivalentes: efeitos do tamanho da classe. Psicologia: Reflexão e Crítica, 2, 233-251.

Haydu, V. B., Omote, L. C., Vicente, P. \& Aggio, N. M. (submetido) Efeitos do tamanho da classe na manutenção de relações de equivalência em um delineamento intra-grupo. Interação em psicologia.

Hübner, M. M. \& Marinotti, M. (2004). Análise do comportamento para a educação: contribuições recentes. São Paulo: ESETEC. 
Kato, O. M., de Rose, J. C., \& Faleiros, P. B. (2008). Topography of responses in conditional discrimination influences formation of equivalence classes. The Psychological Record, 58, 245-267.

Medeiros, J, \& Teixeira, S. (2000). Ensino de leitura e escrita através do pareamento com o modelo e seus efeitos sobre medidas de inteligência. Estudos de Psicologia, 5(1), 181-214.

Omote, L.C, Vicente, P., Aggio, N. M., \& Haydu, V. B. (2009) Manutenção de classes de estímulos equivalentes: um estudo com alunos do Ensino Fundamental. Psicologia: Teoria e Prática, 11, 18-34 Pérez-González, L. A. \& Moreno-Sierra, V. (1999). Equivalence class formation in elderly persons. Psicothema, 11(2), 325-36.

Pinto, A. C. Problemas de memória nos idosos: Uma revisão. (1999) Psicologia, Educação e Cultura, 3(2), 253-295.

Rocha, M. M. (2002). O efeito do número de membros das classes na formação e manutenção da equivalência de estímulos: implicações para o desenvolvimento de estratégias de ensino. Dissertação (mestrado) - Universidade Estadual de Londrina, Londrina.

Saunders, R. R. \& Green, G. (1999). A discrimination analysis of training-structure effects on stimulus equivalence outcomes. Journal of the Experimental Analysis of Behavior, 72, 117-137.

Saunders, R. R., Drake, K. M. \& Spradlin, J. E. (1999). Equivalence class development, retention, expansion and modification in preschool children.
Journal of the Experimental Analysis of Behavior, 71, 195-214.

Saunders, R. R., Wachter, J. \& Spradlin, J. E. (1988). Establishing auditory stimulus control over an eight-member equivalence class via conditional discrimination procedures. Journal of the Experimental Analysis of Behavior, 49, 95-115.

Sidman, M. (1971). Reading and auditory-visual equivalence. Journal of Speech and Hearing Research, 14, 5-13.

Sidman, M. (1994). Equivalence relations: a research story. Boston: Authors Cooperative.

Sidman, M., \& Cresson, O. (1973). Reading and crossmodal tranfer of stimulus equivalence in severe retardation. American Journal of Mental Deficiency, 77, 515-523.

Sidman, M., Kirk, B., Willson-Morris, M. (1985). Six-member stimulus classes generated by conditional discrimination procedures. Journal of the Experimental Analysis of Behavior, 43, 21-42. Sidman, M., \& Tailby, W. (1982). Conditional discrimination vs. mathing to sample: An expansion of testing paradigm. Journal of the Experimental Analysis of Behavior, 37, 5-22.

Yassuda, Batistone, Fortes, \& Néri, (2006). Treino de Memória no Idoso Saudável: Benefícios e Mecanismos. Psicologia: Reflexão e Crítica, 19, 470-481.

Recebido em 20 de julho de 2009 Aceito em 2 de novembro de 2009

Publicação referente ao $2^{\circ}$. Semestre de 2008, impressa em maio de 2011 